\title{
RISQUE DE RÉÉMERGENCE DU PALUDISME AU MAROC ÉTUDE DE LA CAPACITÉ VECTORIELLE D'ANOPHELES LABRANCHIAE DANS UNE ZONE RIZICOLE AU NORD DU PAYS
}

\author{
FARAJ C.*, OUAHABI S.*, ADLAOUI E.*, BOCCOLINI D.**, ROMI R.** \& EL AOUAD R.***
}

Summary: ASSESSMENT OF MALARIA RESURGENCE RISK IN MOROCCO. STUDY OF THE VECTORIAL CAPACITY OF ANOPHELES LABRANCHIAE IN A RICE CULTIVATION AREA IN THE NORTH OF THE COUNTRY

To assess the malaria reintroduction risk in Morocco, we analyzed the malariogenic potential of a rice cultivation area in the north of the country. Our results showed that the receptivity of this area is very high during all the period of the rice cultivation, from May to October. the vectorial capacity of An. labranchiae, malaria vector in Morocco, is considerably high during the summer which corresponds to the rice cultivation period. The risk of autochthonous malaria resumption is important because of the possible presence of gametocytes carriers in the last malaria focus which is bordering the study area. The risk of a tropical malaria introduction is unimportant seen the low vulnerability of the area and the uncertain competence of its vectors considered. However, this risk must be considered with a more attention.

KEY WORDS : Anopheles maculipennis, Anopheles labranchiae, vectorial capacity, receptivity, malaria, risk, Morocco.

I e paludisme a sévi au Maroc pendant des siècles. Il se présentait comme une maladie endémique dans la majorité des provinces du pays. En 1960, un programme national de lutte contre cette maladie a été lancé. Il a permis le contrôle de la situation après 40 ans de lutte acharnée. À partir de 1999, le paludisme se présente sous forme de cas sporadiques à Plasmodium vivax dans quelques foyers résiduels du nord du pays. En 2004, le dernier cas autochtone a été notifié et depuis le Maroc s'efforce de préserver ces acquis par une surveillance vigilante des derniers foyers ; l'objectif étant la certification officielle de l'élimination du paludisme autochtone du Maroc.

Anopheles (Anopheles) labranchiae Falleroni, 1926 a toujours été considéré comme le vecteur principal. Cette espèce qui colonise une très grande partie du pays trouvait sa zone de prédilection dans les plaines atlantiques au nord-ouest où elle constituait plus de

\footnotetext{
* Laboratoire d'Entomologie Médicale, Institut National d'Hygiène, 27, Avenue Ibn Batouta, Agdal 11400, Rabat, Maroc.

** Istituto Superiore di Sanità, viale Regina Elena, 299/00161, Rome, Italie.

***: Institut National d'Hygiène, 27, Avenue Ibn Batouta, Agdal 11400, Rabat, Maroc.

Correspondance : Chafika Faraj - Tél. : 00212377719 02/65.

E-mail : c.faraj@menara.ma, chafikaf@gmail.com
}

\section{Résumé :}

Dans le but d'estimer le risque de réintroduction du paludisme au Maroc, nous avons analysé le potentiel paludogène d'une zone rizicole au nord du pays. Nos résultats ont montré que la capacité vectorielle d'An. labranchiae, vecteur du paludisme au Maroc, était particulièrement élevée pendant la période estivale qui correspond à la période de culture du riz. Le risque d'une reprise de la transmission du paludisme autochtone est élevé du fait de l'éventuelle présence de porteurs de parasite dans le dernier foyer de paludisme limitrophe de la zone d'étude. Le risque d'une introduction du paludisme tropical est faible, vu la faible vulnérabilité de la région et la compétence de ses vecteurs, considérée comme faible. Toutefois, ce risque doit faire l'objet d'une grande attention.

MOTS CLÉS : Anopheles maculipennis, Anopheles labranchiae, capacité vectorielle, réceptivité, paludisme, risque, Maroc.

$95 \%$ du peuplement anophélien (Gaud et al., 1950). Les épidémies les plus meurtrières dans l'histoire du paludisme au Maroc étaient enregistrées dans cette région où An. labranchiae était impliqué dans la transmission aussi bien de $P$. vivax que de $P$. faciparum et de P. malariae (Gaud et al., 1949; Anonyme, 1990). Il proliférait dans les zones marécageuses et les rizières qui constituaient les principaux gites de la région. Dès le lancement du programme de lutte antipaludique, la majorité des zones marécageuses a disparu suite aux grands travaux d'assainissement entrepris. Les campagnes de lutte par utilisation du DDT poursuivies pendant plusieurs années ont limité la prolifération d'An. labranchiae dans les rizières (Houel \& Donadille, 1953; Houel, 1954). En parallèle, le dépistage actif et le traitement des cas ont permis de neutraliser le réservoir du parasite. En 1995, le paludisme autochtone avait déjà disparu de ces zones.

Toutefois, l'extension progressive des rizières dans cette zone extrêmement réceptive et le déclin de la vigilance vis-à-vis de ces biotopes très favorables aux pullulations d'An. labranchiae se sont traduit par un retour offensif de celui-ci ; les densités ont atteint des niveaux inquiétants laissant planer le risque d'une résurgence de la maladie. 
Le présent travail, consacré à l'étude de la réceptivité au paludisme d'une zone rizicole de la province de Larache, a pour objectif de documenter le risque d'une reprise de la transmission dans de telles zones en s'intéressant particulièrement à l'étude de la capacité vectorielle du vecteur.

\section{MATÉRIEL ET MÉTHODES}

\section{ZONE D'ÉTUDE}

L 'étude s'est déroulée dans le nord-ouest du Maroc, dans la localité de Boucharen ( $\left.35^{\circ} 07^{\prime} \mathrm{N}, 06^{\circ} 04^{\prime} \mathrm{O}\right)$ implantée dans une zone rizicole faisant partie de la commune de Louamra située dans le bassin du Loukkos à $17 \mathrm{~km}$ au sud de la ville de Larache. L'élevage des bovins et ovins est développé dans ce village. Les animaux sont parqués dans des abris se trouvant à l'intérieur d'une même enceinte que les habitations humaines. Le climat régnant dans la région est de type méditerranéen semi continental. Les précipitations se répartissent généralement d'octobre à avril avec des pointes en décembre, janvier et février. La pluviométrie moyenne annuelle varie de 600 à $800 \mathrm{~mm}$. Les températures minima extrêmes sont de l'ordre de 1 à $3^{\circ} \mathrm{C}$ en hiver et de 10 à $14^{\circ} \mathrm{C}$ en été. Les températures maxima extrêmes sont de 22 à $26^{\circ} \mathrm{C}$ en hiver et de 34 à $43^{\circ} \mathrm{C}$ en été. Les températures moyennes annuelles se situent autour de $17^{\circ} \mathrm{C}$.

Les rizières sont situées à moins de $300 \mathrm{~m}$ des habitations. Leur submersion est continue, depuis la mise en eau (mai-juin) jusqu'à la récolte (octobre-novembre), engendrant de fortes pullulations d'anophèles dans le village en l'absence de mesure de lutte. Comme méthodes de protection individuelle, les habitants de la localité utilisent des bombes insecticides à usage domestique. Les riziculteurs luttent contre les ravageurs du riz par traitement aérien au malathion (organophosphoré) trois fois durant la période de la culture : au début des phases de tallage, de la montaison et de l'épiaison (coopérative rizicole de Boucharen, communication personnelle).

\section{TAUX D'AGRESSIVITÉ}

L'échantillonnage des populations anophéliennes agressives a été effectué neuf fois d'avril à novembre 2007. Les densités agressives ont été calculées dans deux maisons choisies en fonction de leur situation par rapport aux rizières. L'une située à $300 \mathrm{~m}$ de celles-ci et l'autre $1,3 \mathrm{~km}$. Les moustiques ont été capturés sur sujets humains à l'intérieur et à l'extérieur des habitations du coucher au lever du soleil au rythme de deux jours consécutifs par séance de capture. Quatre captureurs opèrent par nuit; deux à l'intérieur et deux à l'extérieur (soit huit hommes-nuits par séance de cap- ture). Les anophèles appartenant au complexe $A n$. maculipennis ont été identifiés morphologiquement comme décrit par Brunhes et al. (2000) puis par PCR adapté par Proft et al. (1999).

\section{Cycle gonotrophique}

La durée du cycle gonotrophique d'une femelle d'anophèle correspond à l'intervalle de temps séparant deux repas de sang consécutifs. La durée du cycle gonotrophique d'An. labranchiae a été estimée au laboratoire. 48 femelles ont été capturées sur sujets humains et gorgées au moment de la capture. Elles ont été ramenées au laboratoire où elles ont été maintenues individuellement en élevage (température : $25-27^{\circ} \mathrm{C}$; humidité relative : 70-80\%) jusqu'à la ponte. Ces femelles sont mises quelques heures après la ponte puis quotidiennement en contact avec le bras d'un volontaire jusqu'à ce qu'elles acceptent de prendre leur repas de sang. Le cycle gonotrophique est la période qui s'écoule entre deux repas sanguins.

\section{TAUX DE PARTURITÉ}

Le taux de parturité des femelles a été estimé lors de chaque séance de capture par dissection des ovaires et examen de l'aspect des trachéoles selon la méthode de Detinova (1962). Le taux quotidien de survie $p$ a été estimé par la formule de Davidson (1954) :

$$
p=\sqrt[n]{\operatorname{tau} x \text { de parturité }}
$$

où n est la durée du cycle gonotrophique.

\section{INDICE D'ANTHROPOPHILIE}

Les femelles gorgées d'An. labranchiae capturées au repos le matin très tôt dans les chambres à coucher et les abris animaux ont fait l'objet d'une analyse de leur repas de sang pour en identifier l'origine. L'espèce animale sur laquelle s'était gorgé le moustique était déterminée par la technique ELISA selon la méthode décrite par Beier et al. (1988). Les antisérums du bouf, cheval, mouton, chien et homme ont été testés.

\section{CAPACITÉ VECTORIELLE}

La capacité vectorielle représente le nombre moyen de piqûres infectantes que la population d'anophèles peut théoriquement infliger quotidiennement à l'homme à partir d'un cas infectant. Les indices de la capacité vectorielle ont été calculés pour l'ensemble des captures de nuit à partir de la formule de Garret-Jones (1964) dérivée des travaux de Macdonald (1957) :

$$
C V=\frac{m a^{2} p^{n}}{-\ln p}
$$

où :

- ma : densité des femelles agressives capturées au cours de chaque séance de capture. Elle a été établie d'après 


\begin{tabular}{|c|c|c|c|c|c|c|c|c|c|}
\hline \multirow[b]{3}{*}{ Date } & \multicolumn{3}{|c|}{ Piqûres/homme/nuit } & \multicolumn{6}{|c|}{ Piqûres/homme/heure } \\
\hline & \multicolumn{2}{|c|}{ Total } & \multirow[b]{2}{*}{ Moyenne } & \multicolumn{2}{|c|}{$\operatorname{Max}$} & \multicolumn{2}{|c|}{ Min } & \multicolumn{2}{|c|}{ Moyenne } \\
\hline & Intérieur & Extérieur & & Intérieur & Extérieur & Intérieur & Extérieur & Intérieur & Extérieur \\
\hline 16 avril & 2,75 & 1,75 & 2,25 & 2 & 1 & 0 & 0 & 0,27 & 0,17 \\
\hline 16 mai & 15,75 & 10,5 & 13,13 & 7 & 5 & 0 & 0 & 1,57 & 1,05 \\
\hline 30 mai & 31,75 & 15,5 & 23,63 & 16 & 8 & 0 & 0 & 3,17 & 1,55 \\
\hline 19 juin & 158,25 & 173,75 & 166 & 39 & 60 & 2 & 2 & 15,82 & 17,37 \\
\hline 25 juillet & 242,25 & 229,25 & 236 & 71 & 48 & 0 & 0 & 24,22 & 22,97 \\
\hline 14 août & 338,25 & 396,25 & 367 & 71 & 101 & 3 & 5 & 33,82 & 39,62 \\
\hline 4 septembre & 130,25 & 37,75 & 84 & 32 & 15 & 1 & 0 & 13,02 & 3,77 \\
\hline 4 octobre & 51,75 & 62,75 & 57,25 & 14 & 18 & 0 & 1 & 5,17 & 6,27 \\
\hline $1^{\mathrm{er}}$ novembre & 1,5 & 1 & 1,25 & 2 & 1 & 0 & 0 & 0,14 & 0,91 \\
\hline
\end{tabular}

Tableau I. - Variation de la densité agressive d'An. labranchiae à Larache, 2007.

la moyenne des résultats obtenus à l'intérieur et à l'extérieur. Elle est exprimée en nombre de piqûres par homme par nuit.

- $a$ : nombre quotidien de piqûres effectuées sur homme par une femelle d'anophèle. Il représente le produit de la fréquence d'alimentation par l'indice d'anthropophilie.

- In : logarithme népérien

- $p^{n}$ : taux de survie des anophèles pendant les $n$ jours du développement extrinsèque (fonction des températures moyennes quotidiennes relevées sur la zone d'étude).

\section{RÉSULTATS}

\section{FAUNE CULICIDIENNE}

A u total 9495 femelles de culicidés ont été capturées sur homme durant la période de l'étude. Ces captures ont été représentées par les genres Anopheles $(80 \%, \mathrm{n}=7560)$ et Culex $(20 \%, \mathrm{n}=1935)$. Le genre Culex est représenté par les espèces $C x$. theileri Theobald, 1903, Cx. pipiens Linnaeus, 1758, Cx. antennatus, Becker 1903, Cx. univattatus Theobald, 1901 et Cx. laticinctus Edwards, 1913. Le genre Anopheles est représenté uniquement par le complexe d'espèces An. maculipennis. L'identification moléculaire de 193 femelles appartenant à ce complexe a révélé la présence d'une seule espèce : An. labranchiae Falleroni 1926. Cette espèce semble être le seul représentant du complexe au Maroc (Faraj et al. 2004).

\section{TAUX D'AGRESSIVITÉ}

Les valeurs moyennes obtenues par séance de capture sont regroupées dans le tableau I. An. labranchiae a été capturé au cours des neuf enquêtes, avec un pic d'abondance au mois d'août qui correspond à la phase de montaison du riz (367 piqûres par hommes par nuit (PHN)). Les densités les plus basses s'observent en avril avant la mise en eau des rizières et en novembre durant la période de récolte.

\begin{tabular}{lc}
\hline & Durée (jours) \\
\hline Phase 1 (premier repas de sang - ponte) & $4,6 \pm 1,1$ \\
Phase 2 (ponte - deuxième repas) & $0,3 \pm 0,5$ \\
Durée totale & $4,9 \pm 1,1$ \\
\hline
\end{tabular}

Tableau II. - Durée des différentes phases du cycle gonotrophique d'An. labranchiae.

\section{Cycle gonotrophique}

La durée du cycle gonotrophique d'An. labranchiae est de cinq jours. Les résultats sont résumés dans le tableau II.

\section{TAUX DE PARTURITÉ}

Le taux de parturité observé dans la localité de Boucharen varie entre 0,6 et 0,92 (tableau III). Il évolue de façon inverse par rapport aux densités agressives.

\section{INDICE D'ANTHROPOPHILIE}

Nous avons analysé 162 repas sanguins de femelles d'An. labranchiae provenant des chambres à coucher et 198 repas sanguins de femelles capturées dans des abris animaux. An. labranchiae montre une nette préférence pour les bovins : 281 moustiques se sont gorgés sur bœuf dont $56 \%(\mathrm{n}=156)$ ont été collectés dans les abris animaux et $44 \%(n=125)$ dans les chambres à coucher. Onze anophèles seulement se sont gorgés sur homme (tableau IV). Parmi ces 11 femelles, 10 ont été collectées dans les chambres à coucher. Le taux d'anthropophilie moyen parmi les femelles gorgées prises au repos a donc été 0,03 .

\section{CAPACITÉ VECTORIELLE}

Le tableau III montre l'évolution temporelle des différents paramètres de la capacité vectorielle à Larache. L'espérance de vie des femelles d'An. labranchiae varie de 10 à 60 jours. Pendant la saison présumée de transmission, elle est en moyenne de 28 jours. Les estimations des espérances de vie infectantes suggèrent que les femelles vivent assez longtemps après l'âge épidémiologiquement dangereux pour assurer la transmis- 


\begin{tabular}{|c|c|c|c|c|c|c|c|c|}
\hline Date & $\mathrm{ma}$ & $\begin{array}{l}\text { Anophèles } \\
\text { disséqués }\end{array}$ & $\begin{array}{c}\text { Anophèles } \\
\text { pares }\end{array}$ & $\begin{array}{c}\text { Taux } \\
\text { de parturité }\end{array}$ & $\begin{array}{c}\text { Taux } \\
\text { quotidien } \\
\text { de survie }\end{array}$ & $\begin{array}{l}\text { Espérance } \\
\text { de vie }\end{array}$ & $\begin{array}{c}\text { Espérance } \\
\text { de vie } \\
\text { infectante }\end{array}$ & $\begin{array}{c}\text { Capacité } \\
\text { vectorielle } \\
(P . \text { vivax })\end{array}$ \\
\hline $16 / 04$ & 2,25 & 13 & 12 & 0,92 & 0,98 & 59 & 38 & 0,5 \\
\hline $16 / 05$ & 13,13 & 68 & 62 & 0,91 & 0,98 & 52 & 39 & 3,1 \\
\hline $30 / 05$ & 23,63 & 82 & 64 & 0,78 & 0,95 & 20 & 11 & 1,6 \\
\hline $19 / 06$ & 166,00 & 94 & 60 & 0,64 & 0,91 & 11 & 4 & 3,7 \\
\hline $25 / 07$ & 236,00 & 103 & 80 & 0,78 & 0,95 & 20 & 12 & 17,2 \\
\hline $14 / 08$ & 367,00 & 112 & 91 & 0,81 & 0,96 & 23 & 15 & 34,0 \\
\hline 04/09 & 84,00 & 120 & 107 & 0,89 & 0,98 & 42 & 36 & 18,3 \\
\hline $04 / 10$ & 57,25 & 80 & 68 & 0,85 & 0,97 & 30 & 18 & 6,4 \\
\hline $01 / 11$ & 1,25 & 10 & 6 & 0,60 & 0,90 & 10 & 1 & 0,0 \\
\hline
\end{tabular}

Tableau III. - Variation des paramètres entomologiques impliqués dans l'estimation de la capacité vectorielle d'An. labranchiae à Larache, 2007.

\begin{tabular}{cccccccc}
\hline \multirow{2}{*}{$\begin{array}{c}\text { Nombre de } \\
\text { repas analysés }\end{array}$} & Homme & Bovin & Ovin & Chien & Cheval & Mixte & Autres \\
\cline { 2 - 8 } & $3(11)$ & $78(281)$ & $8(29)$ & $2(8)$ & $4(14)$ & $3(10)$ & $2(7)$ \\
\hline 360 & & & &
\end{tabular}

Tableau IV. - Identification de l'origine des repas sanguins d'An. labranchiae à Larache, 2007.

sion d'un hypothétique Plasmodium (4 à 40 jours). La capacité vectorielle pour $P$. vivax a été estimée. Elle est très élevée pendant les mois de juillet, août et septembre. Ceci confère à cette zone une très grande réceptivité au paludisme pendant la période estivale.

\section{DISCUSSION}

L es résultats obtenus montrent que dans une zone anciennement impaludée, la densité du vecteur principal et sa capacité vectorielle ont atteint des niveaux inquiétants. Cette dernière était supérieure, pendant la période allant d'avril à octobre, au seuil critique établi à 0,022 à partir du modèle mathématique de l'épidémiologie du paludisme développé par Molineau et Gramiccia (1980). Nous ne disposons pas de données concernant le niveau de réceptivité initial de cette région pour pouvoir discuter de son évolution. Mais il est clair que l'écosystème de Boucharen offre aux populations d'An. labranchiae des conditions écologiques favorables à leur vie larvaire et imaginale. L'implication des rizières dans la prolifération d'An. labranchiae, aussi bien au Maroc qu'ailleurs, est connue (Houel, 1954; Bettini et al., 1978) et leur implication dans la transmission du paludisme au Maroc a été bien démontrée (Sicault et al., 1935; Gaud et al., 1949; Houel, 1954). Les densités des femelles agressives observées à Boucharen présentent des variations liées aux phases de la riziculture. Elles varient de 2 PHN en avril (avant la mise en eau des rizières) à plus de 350 PHN en août (phase de montaison du riz). Les densités moyennes de femelles agressives sont supérieures à 100 PHN indi- quant une prolifération très importante de ce vecteur dans la région.

À côté d'An. labranchiae, de nombreuses espèces de Culicidae se développent dans ces zones rizicoles. Les moustiques du genre Culex, bien moins abondants que les anophèles, ont été présents pendant toute la période de l'étude et sont considérés comme une source de nuisance par la population.

An. labranchiae, considérée autrefois comme une espèce anthropophile (Sicault et al., 1935; Roubaud, 1935) serait essentiellement zoophile. Cette zoophilie a déjà été mentionnée au Maroc (Houel, 1955; Guy, 1963) et notamment dans la province de Larache (Bailly-Choumara, 1972). Malgré les taux élevés d'agressivité pour l'homme notés au cours de l'étude, les repas sanguins pris sur homme étaient très peu fréquents (3\%). Cette déviation zoophile serait favorisée par la proximité entre le cheptel bovin et les habitations, ainsi que par les mesures de protection individuelle prises par les habitants du village.

Bien que Boucharen offre un biotope favorable au développement de An. labranchiae en matière de disponibilité de gîtes et d'hôtes vertébrés pour les repas de sang, la durée du cycle gonotrophique mesurée en insectarium ne peut donner d'indication sur la durée réelle des phases actives de recherche d'un gîte de ponte et d'un hôte convenable pour le repas de sang. De ce faite, la durée du cycle gonotrophique utilisée dans le calcul de la capacité vectorielle pourrait être sous estimée et par conséquent les valeurs de cette dernière seraient sur estimées.

Les longévités des populations des femelles agressives, mesurées sur une période de six mois par la 
méthode de Detinova (> 10 jours) sont compatibles avec l'achèvement du développement extrinsèque. En fait, $28 \%, 54 \%, 60 \%, 81 \%, 57 \%$ de la population atteindront l'âge épidémiologiquement dangereux respectivement pendant les mois de juin, juillet, août, septembre et octobre.

De façon générale le risque paludogène dans la région de Boucharen est élevé. Il est remarquable pendant les mois de juillet, août et septembre pendant lesquels on note des capacités vectorielles supérieures à 17. En d'autres termes, à partir d'un seul réservoir infectant, ayant passé une nuit du mois d'août à Boucharen on pourrait théoriquement s'attendre à obtenir 34 cas secondaires d'infections palustres. Des estimations analogues de la capacité vectorielle d'An. labranchiae ont été réalisées dans une zone rizicole en Italie. Elles ont donné des résultats comparables (Romi et al., 1977; Romi, 1999).

La réceptivité de la zone est donc très élevée et l'introduction d'un éventuel réservoir de parasite risque de déclencher une épidémie.

En effet, la province de Larache est limitrophe de la province de Chefchaouen, dernier foyer du paludisme autochtone au Maroc, et où la présence de porteurs de parasite est toujours plausible. La contiguité des deux zones et les déplacements de leurs populations contribuent à un risque important d'une reprise de la transmission du paludisme autochtone à $P$. vivax.

Par ailleurs, le risque d'introduction de souches plasmodiales africaines est faible, mais n'est pas à écarter. Le Maroc enregistre chaque année une centaine de cas allochtones à $P$. falciparum dont cinq ont été notifiés dans la province de Larache depuis 1996 (Service des Maladies Parasitaires, Ministère de la santé). En outre, cette région se trouve sur le grand axe routier reliant le Maroc à l'Europe, emprunté par les immigrants originaires des pays au sud du Maroc où le paludisme est endémique. Ces derniers constituent un réservoir de parasite qui échappe à tout contrôle. Bien qu'il ne soit pas certain, qu'An. labranchiae du Maroc est réfractaire aux souches tropicales de P. falciparum, comme cela a été démontré pour An. labranchiae d'Italie (Zulueta et al. 1975; Ramsdale \& Colluzi, 1975), le risque d'une réintroduction du paludisme sud-saharien est une éventualité à redouter.

\section{CONCLUSION}

\footnotetext{
travers cette étude, nous avons étudié la capacité vectrice d'An. labranchiae. Nos résultats 1 ont montré que cette dernière est élevée pendant les mois de juillet, août et septembre. En matière d'évaluation du risque de réémergence du paludisme autochtone, cette observation est à relier, d'une part, avec la proximité de la zone d'étude par rapport à la
}

province de Chefchaouen (dernier foyer de paludisme au Maroc) et, d'autre part, avec la possible circulation de réservoirs de parasites entre ces deux provinces. L'augmentation continue du nombre de cas à $P$. falc $i$ parum importés au Maroc ainsi que la présence d'immigrants en provenance de pays d'endémies échappant à tout contrôle sanitaire constituent également une inquiétude quant à l'introduction du paludisme sud-saharien. Une éventuelle reprise de la transmission du paludisme au Maroc doit être considérée avec une grande attention. De ce fait, il est absolument nécessaire et urgent de veiller à l'application de la réglementation en vigueur concernant l'exploitation des rizières.

\section{REMERCIEMENTS}

ous remercions vivement Mss : M. Elkohli,
L. Laqraa, M. Elrhazi, M. Sbika et A. Arous pour
leur aide technique au laboratoire et sur le terrain. Nous remercions aussi le personnel de la délégation médicale de Larache; les docteurs Bousfiha et Mouzdahir et M. Assebane pour leur soutien et collaboration. Cette étude a reçu le support technique et financier du projet européen EDEN, GOCE-2003010284. Cet article est référencé EDEN103. Le contenu de cette publication est sous la seule responsabilité des auteurs et n'engage pas l'Union Européenne.

\section{RÉFÉRENCES}

AnOnyme. Le paludisme au Maroc : rétrospective et situation actuelle. Bulletin Epidémiologique, 1990, 3, 2-9.

Bailly-Choumara H. Étude détaillée d'une récolte d'Anopheles labranchiae par pièges lumineux portatifs C.D.C (4,5 volts), avec examen de la faune résiduelle, réalisée à Larache, Maroc. Rapport NI0/72, 1972, Laboratoire d'Entomologie, I.S.C. Maroc, 18 p.

Beier J.C., Perkins PV., Wirtz R.A., Koros J., Diggs D., Gargan T.P. \& Koech D.K. Bloodmeal identification by direct enzyme-linked immunosorbent assay (ELISA), tested on Anopheles (Diptera: Culicidae) in Kenya. Journal of Medical Entomology, 1988, 25, 9-16.

Bettini S., Gradoni L., Cocchi M. \& Tamburo A. Rice culture and Anopheles labranchiae in central Italy, 1978, WHO/ $M A L / 78.897$.

Brunhes J., Rhaim A., Geoffroy B., Angel G. \& Hervy J.P. Les moustiques de l'Afrique méditerranéenne. Logiciel d'identification et d'enseignement. Montpellier, France, 2000. IRD \& IPT, CD-Rom collection didactique, Éditions IRD.

DAVIDSON G. Estimation of the survival rate of anophelinae mosquitoes in nature. Nature, 1954, 174, 792-793.

Detinova T.S. Age-grouping methods in dipteral of medical importance with special reference to some vectors of malaria. Genève, Organisation Mondiale de la Santé, série de Monographie, 1962, n 47. 
De Zulueta J., Ramsdal C.D. \& Colluzzi M. Receptivity to malaria in Europe. Bulletin de l'Organisation Mondiale de la Santé, 1975, 52, 109-111.

Faraj C., Adlaoui E., SaAf N., Romi R., Boccolini D., Di Luca M. \& Lyagoubi M. Note sur le complexe Anopheles maculipennis au Maroc. Bulletin de la Société de Pathologie Exotique, 2004, 97 (4), 293-294.

GARRET-Jones C. The human blood index of malaria vectors in relation to epidemiological assessment. Bulletin de l'Organisation Mondiale de la Santé, 1964, 30, 241-261.

Gaud J., Faure F. \& Maurice A. Répartition et fréquence relative des espèces anophéliennes au Maroc. Annales de Parasitologie Humaine et Comparée, 1950, 25, 53-60.

Gaud J., Mechali D. \& Delrieu J. Riziculture et paludisme au Maroc. Bulletin de l'Institut d'Hygiène du Maroc, 1949, 9, 181-190.

GuY Y. Les rapports entre l'anophélisme et le paludisme. Bulletin de la Société des Sciences Naturelles et Physiques, Maroc, 1959, 39, 83-90.

GuY Y. Les Anophèles du Maroc. Mémoires de la Société des Sciences Naturelles et Physiques, Maroc, Zoologie, Rabat, 1959, 7, 235

GuY Y. Renseignements fournis par l'étude du rapport larves adultes d'Anophèles. Annales de Parasitologie Humaine et Comparée, 1962, 37, 633-643.

GuY Y. Bilan épidémiologique du paludisme au Maroc (données recueillies entre 1960, 1961 et 1962). Annales de Parasitologie Humaine et Comparée, 1963, 38, 823-857.

Houel G. \& Donadille F. Vingt ans de lutte antipaludique au Maroc. Bulletin de l'Institut d'Hygiène du Maroc, 1953, 13, 3-51.

Houel G. La lute antipaludique dans les zones rizicoles du Maroc. Bulletin de l'Institut d'Hygiène du Maroc, 1954, NS. Tome XIV, n 1-2, 43-90.

Houel G. Note sur l'orientation trophique de An. labranchiae au Maroc. Bulletin de l'Institut d'Hygiène du Maroc, 1955, $15,387$.

MCDOnald G. The epidemiology and control of malaria. London, Oxford University Press, 1957.

Molineaux I. \& Gramiccia G. Le projet Garki. World Health Organization, Geneva, 1980.

Proft J., MaIER W. \& Kampen H. Identification of six sibling species of the Anopheles maculipennis complex (Diptera: Culicidae) by polymerase chain reaction assay. Parasitology Research, 1999, 85, 837-843.

Ramsdal CD. \& Coluzzi M. Studies on the infectivity of tropical African strains of Plasmodium falciparum to some southern European vectors of malaria. Parasitologia, 1975, 17, 39-48.

Romi R., Pierdominici G., Severini C. Tamburro A. Cocchi M., Menichetti D., Pili E \& Marchi A., Status of malaria vectors in Italy. Journal of Medical Entomology, 1997, 34, 263-271.

Romi R. Anopheles labranchiae, an important malaria vector in Italy, and other potential malaria vectors in Southern Europe. European Mosquito Bulletin, 1999, 4, 8-10.

Roubaud E. Variété nouvelle de l'Anopheles maculipennis au Maroc, An. maculipennis sicaulti (n. var.). Bulletin de la Société de Pathologie Exotique, 1935, 28, 107-111.
Sicault G., Messerlin A., Lumeau J. \& Fritz J. Le paludisme dans le Gharb. Bulletin de l'Institut d'Hygiène du Maroc, 1935, 5, 5-91.

Reçu le 26 février 2008 Accepté le 7 mai 2008 